\title{
A Variable Stiffness Robotic Probe for Soft Tissue Palpation
}

\author{
Nicolas Herzig ${ }^{1}$, Perla Maiolino ${ }^{2}$, Fumiya Iida $^{2}$ and Thrishantha Nanayakkara ${ }^{1}$ RAL version
}

\begin{abstract}
During abdominal palpation diagnosis, a medical practitioner would change the stiffness of their fingers in order to improve the detection of hard nodules or abnormalities in soft tissue to maximise haptic information gain via tendons. Our recent experiments using a controllable stiffness robotic probe representing a human finger also confirmed that such stiffness control in the finger can enhance the accuracy of detecting hard nodules in soft tissue. However, the limited range of stiffness achieved by the antagonistic springs variable stiffness joint subject to size constraints made it unsuitable for a wide range of physical examination scenarios spanning from breast to abdominal examination. In this paper, we present a new robotic probe based on a variable lever mechanism (VLM) able to achieve stiffness ranging from $0.64 \mathrm{~N} . \mathrm{m} / \mathrm{rad}$ to $1.06 \mathrm{~N} . \mathrm{m} / \mathrm{rad}$, that extends the maximum stiffness by around 16 times and the stiffness range by 33 times. This paper presents the mechanical model of the novel probe, the Finite Element (FE) simulation as well as experimental characterization of the stiffness response for lever actuation.
\end{abstract}

Index Terms-Compliant Joint/Mechanism, Force and Tactile Sensing, Haptics and Haptic Interfaces, Medical Robots and Systems, Biomimetics.

\section{INTRODUCTION}

D URING the last decades, studies on medical palpation have been widely addressed in the literature. Indeed, this medical gesture consists in an examination of a patient body with the fingers or hands to evaluate the stiffness of the patient tissue. Palpation is used for global diagnosis with abdominal or thoracic palpation or during an open surgery to detect the position of a hard nodule. With the improvement done in the robotic, medical and biomedical fields, several robot and devices for medical palpation have been developed. These robots and devices can be classified into two categories: the probes and the haptic rendering devices.

The probes are mechanical element designed to perform the palpation in the role of the physician. These devices integrate

Manuscript received: September 7, 2017; Revised December 6, 2017; Accepted December 29, 2017.

This paper was recommended for publication by Editor Nikos Tsagarakis upon evaluation of the Associate Editor and Reviewers' comments. This work was funded by The United Kingdom Engineering and Physical Sciences Research Council (EPSRC) MOTION grant [EP/N03211X/2]

$1 \mathrm{~N}$. Herzig and T. Nanayakkara are with the Dyson School of Design Engineering, Imperial College London, South Kensington SW7 1NA London, United Kingdom. n.herzigeimperial.ac.uk and t.nanayakkara@imperial.ac.uk

2 P. Maiolino and Fumiya Iida are with the Engineering Department, University of Cambridge, Trumpington Street, Cambridge CB2 1PZ United Kingdom. pm640@cam.ac.uk and fi224@cam.ac.uk

This paper has supplementary downloadable material available at http://ieeexplore.ieee.org.

Digital Object Identifier (DOI): see top of this page. sensors and actuators to proceed the palpation and measure the reaction of the soft tissues of the patient. In particular, several robotic probes for palpation have been developed to detect hard nodules in soft tissue mainly to localize tumor [1] during Minimally Invasive Surgery (MIS) [2]. For instance, in previous work, we have shown the interest of stiffness variation to estimate the depth of a hard nodule in soft tissue palpation [3]. Indeed, based on an antagonistic springs variable stiffness joint, we have developed a controller which maximize the information gain for estimating the depth of a stiff inclusion. Similarly, Talasaz and Patel have developed a tactile probe for MIS with a hybrid impedance controller to estimate the depth of a stiff inclusion inside soft tissues [4]. This method gives good results but does not show what is the range of stiffness used to obtain a good estimation of the depth of the stiff inclusion. More examples of suitable probes for nodule detection in MIS are described in [5]. The main limitations of these probes are the force and the stiffness ranges. Since they are designed to be used inside the patient body, most of them are small and not suitable for external palpation. However, only a few robotic palpation probes have been designed for external examination that varies from soft tissues like the breast [6], [7] to harder tissues like the abdomen. According to the authors' knowledge, only one palpation probe has been developed for abdominal palpation [8] which is a one Degree of Freedom (DoF) system actuated by cables able to measure the stiffness of the tissue using a position sensor and a force sensor. Unfortunately, this robotic palpation probe cannot change the stiffness to improve information gain as highlighted in [3].

Furthermore, tactile and haptic feedback devices have been developed to study or teach the medical palpation. Indeed, these devices can be either phantom to mimic the soft tissue behavior [9] or some small actuators which can be integrated to a tool handle or a robot telemanipulator to give feedback to a surgeon during MIS. Various technology has been used to reproduce the human tissues behavior as pneumatic actuation, granular jamming [10], vibrotactile actuation [11] and passive or active Variable Stiffness Actuators (VSA) [12]. Due to the complexity of studying in vivo medical gestures, these devices are suitable to collect data and understand how the physicians perform the palpation. The phantoms or simulators are also interesting tools to learn or practice medical gesture in a riskfree environment.

During a robotic palpation, it is required to control the robot compliance to avoid any damage to itself, to the patient, or the environment. In the last three decades, several approaches have been used to integrate a compliant behavior in robotics. 
In particular, they can be distinguished in two main categories: controllers and actuators.

The first category is based on a control approach and it is related to the development of stiffness or impedance controllers which can be either linear [13] or for some specific applications, like pneumatic or hydraulic robots, nonlinear [14], [15]. If those controllers can be implemented on robots without particular hardware modifications, a force/torque sensor or observer is often needed to improve the controllers' performance. The fact that these controllers often do not store energy constitutes the main limitation of this approach for dealing with environmental disturbances given limited controller bandwidth [16].

The second approach mainly concerns developing dedicated actuators with embedded passive stiffness or damping components. Those actuators are commonly called VSA or Variable Impedance Actuators (VIA). According to Van Ham et al. [17], those VSAs can be distinguished in four categories: equilibrium-controlled stiffness [18], antagonistic-controlled stiffness [19], structural-controlled stiffness [20] and Mechanically Controlled Stiffness [21]. In this paper, a new robotic abdominal palpation probe (see Fig. 1) with a variable stiffness joint is presented. This probe, called VLM probe, is based on a Variable Lever Mechanism (VLM) and is designed to perform abdominal palpation with variable stiffness. This stiffness variation simplifies the control strategy for the patient/robot interaction, but also should improve the localization and depth estimation of the abdominal organs [22]. The robotic palpation probe described in this paper can be considered as a structuralcontrolled stiffness joint.

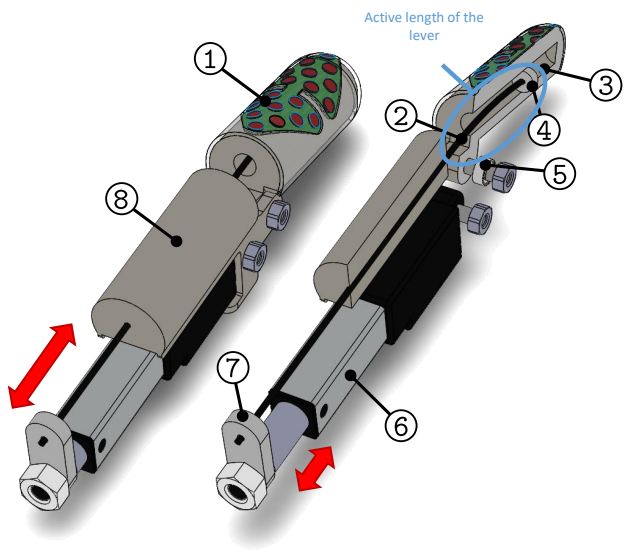

Fig. 1: Design of the palpation probe. The Tip link and the tactile sensor are cut to show the carbon rod and PTFE cylinder inside the Tip link on the right part of the figure. The configurations show the lever bending due to an external force applied on the Tip link for two different active lengths of the carbon rod. Parts: 1: Tactile sensor (Cyskin), 2: Carbon rod, 3: Tip link, 4: PTFE cylinder, 5: Ball bearing, 6: Linear actuator, 7: Link actuator-carbon rod, 8: Base link.

As for most of the VLM mechanisms described in the literature, Awad et al. proposed the pVSJ, a variable stiffness joint with a variable lever mechanism which applies forces on two springs [23] (torsional in that particular case). The pVSJ has a large range of stiffness (theoretically from $0 \mathrm{Nm} / \mathrm{rad}$ to more than $1000 \mathrm{Nm} / \mathrm{rad}$ ) for a variable lever length from $0 \mathrm{~mm}$ to $30 \mathrm{~mm}$, but the design approach does not allow miniaturization to a human finger size. Moreover, the fact that a small variation of the lever length implies a significant change of stiffness, it also needs an accurate position control for the lever actuation.

The main contributions of this paper are related to the variable stiffness probe design, subject to size constraints, and the study performed to understand how the stiffness can be controlled to perform palpation with different compliance levels.

\section{Materials And Methods}

\section{A. The VLM probe}

As shown in Fig. 1, the VLM probe is composed of two main 3D printed parts (Tip link and Base link) connected by a revolute joint. The compliant behavior is obtained by a $1.5 \mathrm{~mm}$ diameter carbon rod that slides inside the two links. Indeed, when an external force is applied to the Tip link, the latter rotates around the joint and the carbon rod bends. The stiffness of the joint depends on the length of the carbon rod inside the Tip link (active length of the carbon rod) due to the cantilever effect. This mechanism offers significant advantages to extend the stiffness range subject to size constraints compared to our previous work based on an antagonistic spring loaded joint [22]. An Actuonix L12-30-50-6-I linear actuator is used to control the active length of the carbon rod. A $6.2 \mathrm{~mm}$ diameter Polytetrafluoroethylene (PTFE) cylinder is added to the carbon rod tip to reduce friction with the Tip link. The association of the carbon rod and the PTFE cylinder is seen as a lever. In the rest of the paper, the active length of the carbon rod or lever length will be used equally.

The stiffness and force ranges of the new probe are based on the recent work done in abdominal and breast palpation [7], [8].

A capacitive tactile sensor based on the CySkin architecture [24] is mounted on the Tip link to mimic cutaneous perception of human fingertips. This sensor covers an area of about 780 $\mathrm{mm}^{2}$ with 20 taxels. An ATI NANO25 6-axis Force/torque sensor was mounted at the base of the probe to mimic kinesthetic force feedback obtained from the base of the finger. This sensor is placed at the base of the probe to represent the proprioception sensing function of a tendon and muscle organs.

This probe mechanism and the sensor arrangement allows us to conduct experiments to understand how the variable stiffness joint affects the quality of perception in the two sensor modalities.

\section{B. Probe stiffness characterization setup}

In order to analyze the stiffness variation of the palpation probe depending on the lever length, a dedicated test bench have been designed as shown in Fig. 2.

During the experiment, the Aerotech ANT130-XY stage applies an angular displacement to the Tip link of the probe. Then 


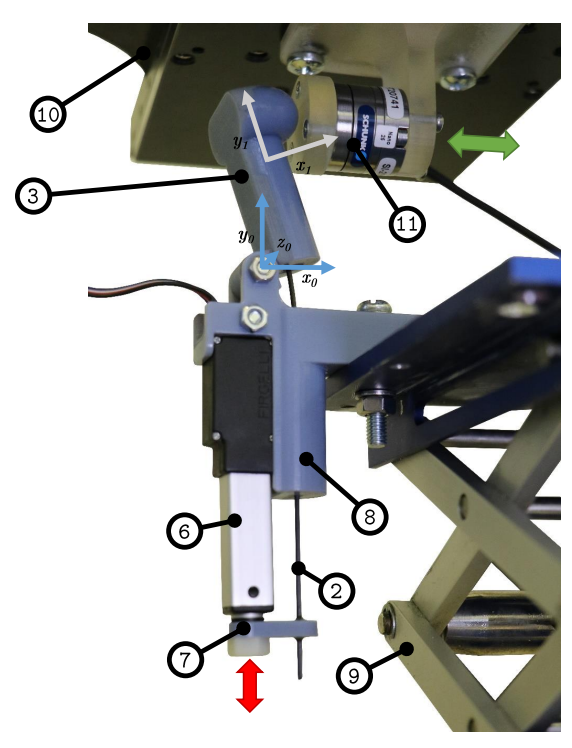

Fig. 2: Palpation probe and test bench. Parts: 2: Carbon rod, 3: Tip link, 6: Linear actuator, 7: Link actuator-carbon rod, 8: Base link, 9: Laboratory lift, 10: XY stage, 11: Force/Torque sensor.

the force applied by the Tip link on the XY stage is measured with a ATI NANO25 Force/torque sensor. A Labview code acquired force and displacement data at $1000 \mathrm{~Hz}$ sampling frequency via two National Instruments cards (NI USB 6341 for acquiring and controlling the linear actuator of the probe, and a NI PCIe 6320 for the force/torque data acquisition).

The Tip link and Base link geometries have been slightly adapted to simplify the vertically mounted experimental study. A torus extruded geometry was added on the upper part of the Tip link and a cuboid extrusion was added to the Base link. The geometry modifications have been done to ensure that the contact between the probe and the force sensor is applied on a single point and to simplified the probe fastening to the laboratory lift. In order to simplify the implementation, the tactile sensor has not been integrated on the test bench Tip link. The aim of the test bench is to study the VLM probe stiffness behavior and the tactile sensor does not affect the stiffness variation of the probe.

\section{Palpation test}

Fig. 3 shows the VLM probe in a palpation experiment. The VLM probe can move in the three Cartesian directions thanks to the XY stage and a linear actuator added on the $\mathbf{z}$ axis (not shown in the figure). The phantom underneath the probe is made of Ecoflex 00-10 silicone. The latter is $148 \mathrm{~mm}$ long, $100 \mathrm{~mm}$ wide and $28 \mathrm{~mm}$ thick. A stiff inclusion with a diameter of $16 \mathrm{~mm}$ has been introduced at a depth of $2 \mathrm{~mm}$ from the top of the phantom.

\section{Mechanical Model}

We conducted experiments to quantify the equivalent stiffness of the revolute joint as a function of the active length of the carbon rod. It is assumed that the only deformable body

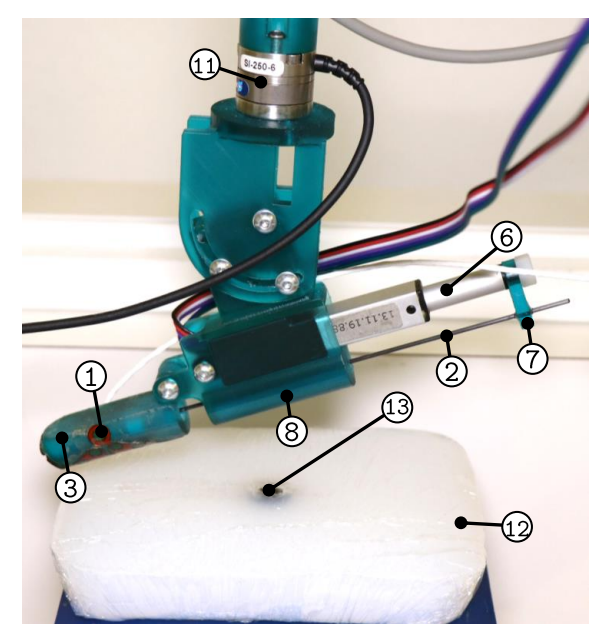

Fig. 3: Palpation of a silicone phantom with the VLM probe. Parts: 1: Tactile sensor (Cyskin), 2: Carbon rod, 3: Tip link, 6: Linear actuator, 7: Link actuator-carbon rod, 8: Base link, 11: Force/Torque sensor, 12: Silicone phantom, 13: Stiff inclusion.

in the probe is the carbon rod and that the latter behaves as cantilever beam of length equal to the lever length. The joint friction and weights of the links are neglected because they are very small compared to the other forces involved. It has to be noticed that in this paper, only the static analysis is studied.

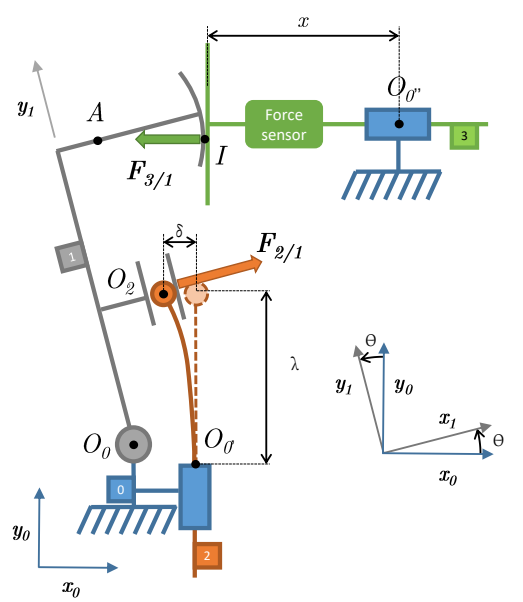

(a)

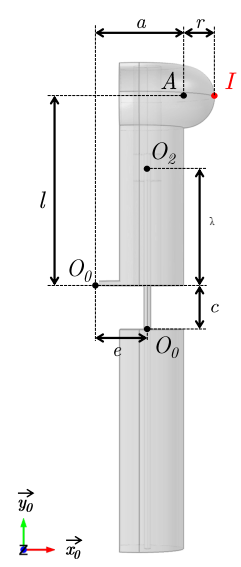

(b)
Fig. 4: Kinematic model. (a) Kinematic scheme. Dashed lines refers to the carbon rod position when no force is applied. (b) Kinematic parameters.

Fig. 4a shows an equivalent kinematic scheme of the probe. That scheme illustrates the different bodies, the bodies 0 , 1, 2, 3 refer to the Base link, the Tip link, the lever and the XY stage with the force sensor respectively. The main kinematic parameters and forces are also illustrated on Fig. 4. The parameters are defined as follows:

$$
\begin{array}{ll}
\mathbf{0 A}=a \mathbf{x}_{\mathbf{1}}+l \mathbf{y}_{\mathbf{1}}, & \mathbf{O}_{\mathbf{0}} \mathbf{O}_{\mathbf{0}^{\prime}}=e \mathbf{x}_{\mathbf{0}}-c \mathbf{y}_{\mathbf{0}} \\
\mathbf{A I}=r \mathbf{x}_{\mathbf{0}}, & \mathbf{O}_{\mathbf{0}^{\prime}} \mathbf{O}_{\mathbf{2}}=-\delta \mathbf{x}_{\mathbf{0}}+\lambda \mathbf{y}_{\mathbf{0}} \\
\mathbf{O}_{\mathbf{0}^{\prime \prime}} \mathbf{I} \cdot \mathbf{x}_{\mathbf{0}}=-x, & \mathbf{O}_{\mathbf{0}} \mathbf{O}_{\mathbf{0}^{\prime \prime}} \cdot \mathbf{x}_{\mathbf{0}}=r+a \\
\mathbf{O}_{\mathbf{0}} \mathbf{O}_{\mathbf{2}} \cdot \mathbf{x}_{\mathbf{1}}=e &
\end{array}
$$


Where $\lambda$ is the lever length and $\delta$ is the deflection of the latter. $\theta$ is the angle of the revolute joint between the Base and Tip links and $x$ is the displacement applied by the $\mathrm{XY}$ stage to the Tip link. The position of $O_{0^{\prime \prime}}$ is chosen in order to have for $x=0, \theta=0$. Each force is denoted as $\mathbf{F}_{\mathbf{i} / \mathbf{j}}$ and refer to the vector of the force applied by the link $i$ on the link $j$. One can notice that the contact between the Tip link and the PTFE cylinder is modeled as a single contact point. Indeed even if the PTFE part is a cylinder, it is assumed that the length of that cylinder, and then the contact area with the Tip link, are small. This assumption has been taken in order to simplify the analysis.

\section{A. First model: Beam spring behavior}

A simple approximation could be to assume that the angle $\theta$ of the probe is equal to the curvature of the cantilever free end. Secondly by assuming that the equivalent torque $\mathbf{M}_{\mathbf{3 1}}$, generated by the force $\mathbf{F}_{\mathbf{3} / \mathbf{1}}$ at $O_{0}$ is directly applied to the carbon rod, the behavior of the probe can be seen as a beam spring with a length $\lambda$. With those assumptions, the relation between $M_{31}$ and $\theta$ comes:

$$
\theta=\frac{M_{31} \lambda}{E I_{x}}
$$

where, $E$ is the Young modulus of the carbon rod, $M_{31}$ the norm of the equivalent torque generated by the force $\mathbf{F}_{\mathbf{3} / \mathbf{1}}$ at $\mathrm{O}_{0}$ :

$$
M_{31}=-F_{31}(a \sin \theta+l \cos \theta),
$$

and $I_{x}$ is the second moment of area of the rod in respect to $\left(\mathrm{O}_{0^{\prime}}, \mathbf{x}_{\mathbf{0}}\right)$ axis given by

$$
I_{x}=\frac{\pi d^{4}}{64}
$$

where, $d$ is the diameter of the carbon rod and $\mathbf{F}_{\mathbf{3} / \mathbf{1}}=F_{31} \mathbf{x}_{\mathbf{0}}$ (n.b. $F_{31}<0$ on Fig. 4a). The equivalent angular stiffness of the variable stiffness probe is given by

$$
K_{\theta}=\frac{d M_{31}}{d \theta} .
$$

From (2) it comes that:

$$
K_{\theta}=\frac{E I_{x}}{\lambda} .
$$

Equation (6) gives a first simple model to describe the angular stiffness of the probe for different lengths of the lever. Unfortunately, this model, commonly used for describing the stiffness of a torsional spring made with a beam [23], is not accurate enough to describe the real behavior observed experimentally ( $c f$. section $\mathrm{V}$ ). The next subsection gives a mechanical study to obtain a more accurate model.

\section{B. Second model: A model from kinematic and static approach}

Two closed chains of the kinematic model subject to constraint equations in (7) and (8) are solved to obtain a second model.

$$
\begin{gathered}
x=a(1-\cos \theta)+l \sin \theta, \\
(e-\delta) \cos \theta+(\lambda-c) \sin \theta=e .
\end{gathered}
$$

Equation (7) gives the mathematical relation between the displacement applied by the XY stage $x$ and the angle of rotation of the revolute joint $\theta$. Equation (8) illustrates the link between the angle $\theta$ and the deflection of the carbon rod $\delta$.

As explained previously, a static analysis is performed to obtain the relation between the stiffness of the probe and the active length of the carbon rod. Thus at equilibrium, the net torque of the body 1 computed at $O_{0}$ follows the relationships in (9)

$$
M_{31}+((e-\delta) \sin \theta+(\lambda-c) \cos \theta) F_{21}=0,
$$

where, $\mathbf{F}_{\mathbf{2} / \mathbf{1}}=F_{21} \mathbf{x}_{\mathbf{1}}$.

The carbon rod is modeled as a cantilever beam of length $\lambda$ (the active length). Indeed the design of the cylindrical cut in the Tip link, where the carbon rod and the PTFE cylinder can slide, have been studied to allow only one contact at the end of the lever. On the other hand, the cylindrical cut in the Base link is assumed to be designed to allow the translation of the rod along the longitudinal axis of the rod but to constrain the radial movement of the latter. Then the Euler-Bernoulli beam theory for a cantilever beam with a point load on the free end of the beam leads to (10) which gives a relation between the force applied by the carbon rod on the Tip link and the deflection of the rod.

$$
F_{21}=\frac{3 E I_{x} \delta}{\lambda^{3}}
$$

It has to be noticed that from the definition given in (1), $F_{21}>0$ implies $\delta>0$.

By substituting $\delta$ and $F_{21}$ in (9) by the expression obtained in (8) and (10), $M_{31}$ can finally be computed as:

$$
\begin{aligned}
& M_{31}=3 E I_{x}\left(\frac{(\lambda-c)((\cos \theta-1) e+(\lambda-c) \sin \theta)}{\lambda^{3}}\right. \\
& \left.+\frac{((\lambda-c) \sin \theta-e)((\cos \theta-1) e+(\lambda-c) \sin \theta)}{\lambda^{3} \cos \theta}\right) .
\end{aligned}
$$

The equivalent stiffness of the probe can now be computed by differentiation as shown in (5). The non linear result obtained is given by:

$$
\begin{aligned}
& K_{\theta}=-\frac{3 E I_{x}}{2 \lambda^{3} \cos (\theta)^{3}}\left(6 c \lambda+c^{2} \cos (2 \theta)+e^{2} \cos (2 \theta)\right. \\
& +\lambda^{2} \cos (2 \theta)-3 c^{2}-3 e^{2}-3 \lambda^{2}+2 e^{2} \cos (\theta) \\
& -8 c e \sin (\theta)+8 e \lambda \sin (\theta)-2 c \lambda \cos (2 \theta)+c e \sin (2 \theta) \\
& -e \lambda \sin (2 \theta)) .
\end{aligned}
$$

To simplify the analysis, $K_{\theta}$ is linearized around $\theta=0$ since the angular displacement of the probe is small for most palpation tasks. After linearization $K_{\theta}$ can be written as:

$$
K_{\theta}=3 E I_{x} \frac{(\lambda-c)(\lambda-c+3 e \theta)}{\lambda^{3}} .
$$

Equation (13) shows that the stiffness depends on the lever length $\lambda$. The model is fitted and compared to the experimental results in section $\mathrm{V}$. It can be also noticed that the obtained model can help to design the probe by changing the dimensional parameters of the probe such as the diameter of the carbon rod, which changes the second moment of area $I_{x}$, or the range of the active length of the carbon $\operatorname{rod} \lambda$. 


\section{Simulation}

In order to validate the rationale behind the probe design, a Finite Element (FE) analysis of the variable stiffness probe behavior has been performed using COMSOL multiphysics, Fig. $4 \mathrm{~b}$ shows the geometrical model of the probe used for performing the FE simulations. Table I gives the parameters used for the simulations.

TABLE I: Simulation parameters

\begin{tabular}{cccccc}
\hline \hline Parameter & Value & Unit & Parameter & Value & Unit \\
$a$ & 20 & $\mathrm{~mm}$ & $c$ & 10 & $\mathrm{~mm}$ \\
$d$ & 1.5 & $\mathrm{~mm}$ & $e$ & 11.8 & $\mathrm{~mm}$ \\
$r$ & 7 & $\mathrm{~mm}$ & $l$ & 43 & $\mathrm{~mm}$ \\
$E$ & 102 & $\mathrm{MPa}$ & $\nu$ & 0.49 & \\
\hline \multicolumn{2}{c}{ Variable Parameter } & & $22: 2: 52$ & $\mathrm{Cnit}$ \\
$\lambda$ & & $0: 0.25: 5$ & $\mathrm{~N}$ \\
$F_{31}$ & & &
\end{tabular}

A stationary study has been performed applying a point force in $\mathrm{x}_{\mathbf{0}}$ direction on a defined point of the toroid evaluating the obtained displacement. The simulation has been performed for different carbon fiber lengths $\lambda$ in the range from $22 \mathrm{~mm}$ to $52 \mathrm{~mm}$ by $2 \mathrm{~mm}$ steps and applied forces $F_{31}$ in the range from $0 \mathrm{~N}$ to $-5 \mathrm{~N}$ by $-0.25 \mathrm{~N}$ steps in the $\mathrm{x}_{0}$ at the point $I$ (in red on Fig. 4b). The boundary conditions have been defined as follows:

1) The displacements and rotations of the bottom surface of the Base link are constrained in all directions.

2) the displacements of the $O_{0}$ point are constrained in all directions.

It has to be noticed that for this simulation the material is assumed to be isotropic and homogeneous. it is also assumed that the materials follow the Hooke's law, i.e., only the linear elasticity of the materials are taken into account.

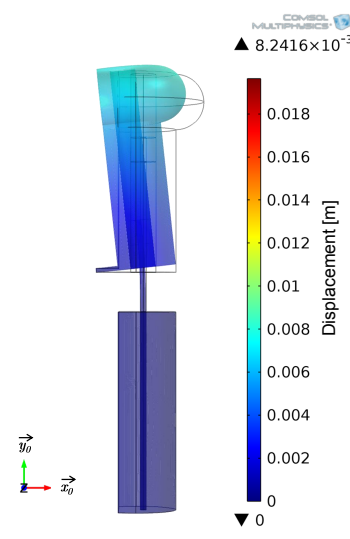

(a)

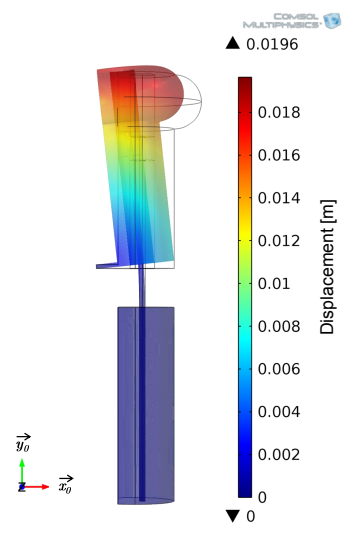

(b)
Fig. 5: FE simulations performed with COMSOL multiphysics. (a) The simulated displacement of the Tip link due to an applied force of $F_{31}=-5 \mathrm{~N}$ and with a $\lambda=22 \mathrm{~mm}$ carbon fiber length. (b) The simulated displacement of the Tip link due to an applied force of $F_{31}=-5 \mathrm{~N}$ and with a $\lambda=44$ $\mathrm{mm}$ carbon fiber length.
Fig. 5a and Fig. 5b present the simulated displacement of the Tip link when a force $F_{31}=-5 \mathrm{~N}$ is applied with a carbon fiber length $\lambda=22 \mathrm{~mm}$ and $\lambda=44 \mathrm{~mm}$ respectively. As expected this figure shows that the displacement of the Tip link nodes is proportional to the distance between the nodes and the point $O_{0}$. By comparing the two figures, it can be noticed that the displacement is higher for a longer carbon rod lever.

Fig. 6 gives the results of all the simulations proceeded, the equivalent torque $M_{31}$ for the different angular displacement $\theta . M_{31}$ is computed from the expression (3) and theta is estimated by the linearization of (7) around the point $\theta=0$. That linearization gives:

$$
\theta \approx \frac{x}{l}
$$

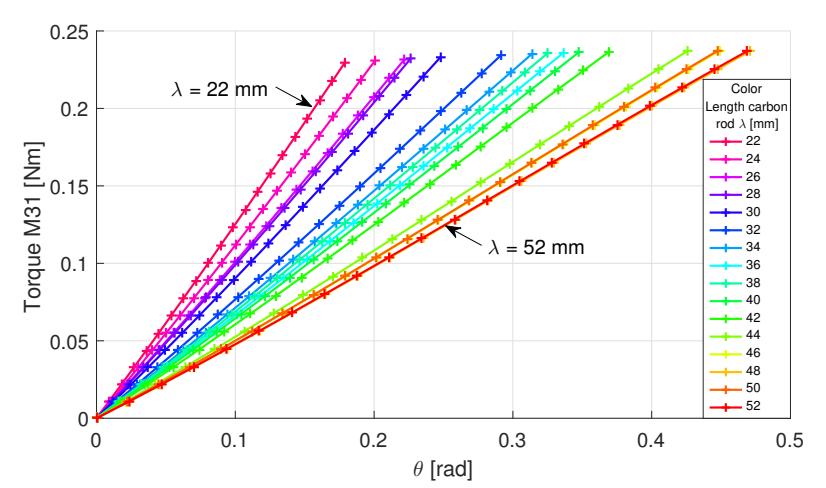

Fig. 6: FE simulation results: Torque $M_{31}$ as a function $\theta$ for 16 different lever length $\lambda$.

Fig. 6 shows that for each active length of the carbon rod, the torque is nearly proportional to the angular displacement. From those simulations, the equivalent stiffness of the variable stiffness probe as a function of the lever length can be studied. Indeed, it can be deduced from (5) that for a given active length $\lambda$ the equivalent stiffness of the probe $K_{\theta}$ is the slope of the curve $M_{31}=f(\theta)$. The equivalent stiffnesses $K_{\theta}$ obtained with the simulation are compared to the experimental results in the next section.

\section{EXPERIMENTAL RESUltS}

This section presents the experimental results obtained with the setup described in section II-B. In the experiment, the $\mathrm{XY}$ stage applies a linear displacement $x$ to the Tip link. The displacement is applied in steps of $0.5 \mathrm{~mm}$ from $0.5 \mathrm{~mm}$ to $10.5 \mathrm{~mm}$. The force applied by the XY stage on the Tip link $F_{31}$ is then measured by the ATI 6-axis force/torque sensor. In order to reach the steady state of the force, each step is separated by 2 seconds. Then when all displacement steps have been applied, the lever length is modified and the experiment starts again. The active carbon rod length $\lambda$ has been set from $22 \mathrm{~mm}$ to $52 \mathrm{~mm}$ in $2 \mathrm{~mm}$ steps for 3 trials, and then set from $52 \mathrm{~mm}$ to $32 \mathrm{~mm}$ in $-2 \mathrm{~mm}$ steps for 3 other trials. The first 3 trials are denoted from 1 to 3 and the second 3 trials will be denoted from 4 to 6 . The first part of the supplementary video 

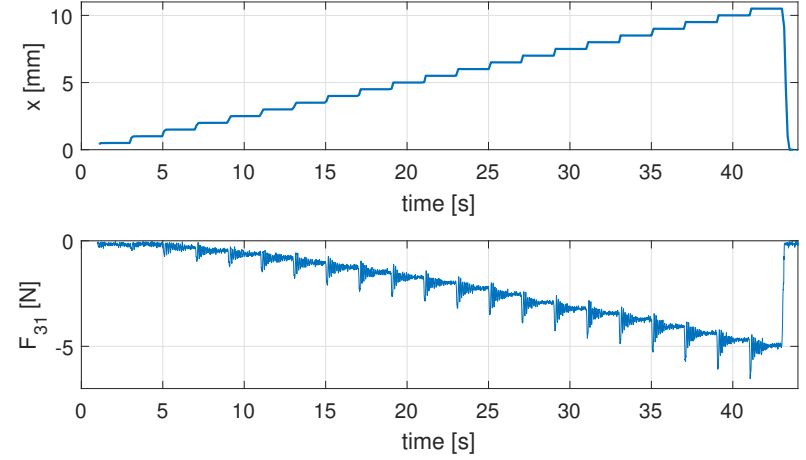

Fig. 7: Force $F_{31}$ and linear displacement $x$ measured during the sixth trial with the carbon rod active length $\lambda=22 \mathrm{~mm}$.

shows a part of the stiffness characterization experiment and some raw data acquired.

An example of the raw data measured during one trial is shown in Fig. 7. This figure shows that higher the displacement is, the higher the reaction of the probe is (the reaction force is equal to $\left.-F_{31}\right)$. It can be seen that each displacement step induces a force peak. That phenomenon is due to the damping of the carbon rod. In this study, only the static behavior of the probe is considered. The average force at steady state is computed for each step of displacement, for each trial, and each lever length. Then from (3) the torque $M_{31}$ is computed. As in the previous section $\theta$ is computed from (14).

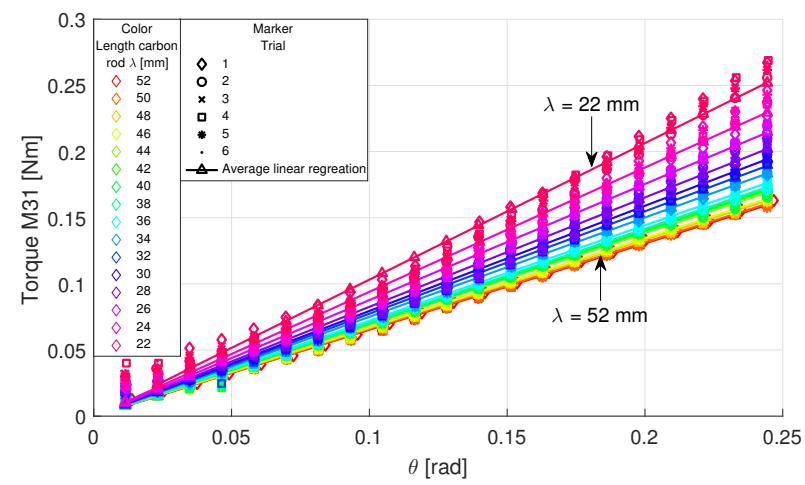

Fig. 8: Torque $M_{31}$ as a function $\theta$ for 6 trials and 16 different lever lengths $\lambda$. Markers numbered from 1 to 6 are related to each experimental trial. Solid lines with triangle markers give the average linear regression obtained for each lever length.

Fig. 8 illustrates the relation between the angular displacement $\theta$ and the torque $M_{31}$ for different lengths of the lever. It can be noticed that the stiffness is linear for higher $\lambda$ values with a gradual tendency to exhibit a nonlinear stiffness variation for lower $\lambda$.

For each trial at a given lever length, a linear regression is applied (the $R^{2}$ value for all regression are higher than 0.98). Fig. 9 shows the gradients obtained for the corresponding regressions. The latter shows that the equivalent stiffness of the probe decreases for increasing lever length. The equivalent stiffnesses computed from the FE simulation results are also given in Fig. 9. As one can see, the FE gives a rough estimate of the experimental results. The differences between the ex-

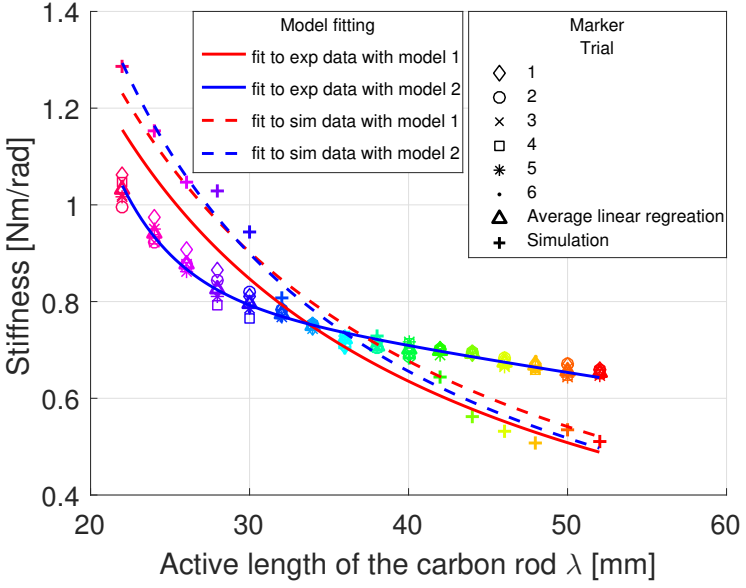

Fig. 9: Equivalent stiffness of the carbon rod as a function of the active length of the carbon rod. Markers numbered from 1 to 6 are related to each experimental trial. Triangle markers show for each lever length the average stiffness computed from experimental results. Plus sign markers refer to the stiffness computed from the FE simulation results. Solid lines are models fitted to the experimental data, and dashed lines are those fitted to the FE simulation results. The red curves are obtained by fitting the model given by (15) whereas the blue curves are obtained by fitting the model given by (16).

periment and simulation can be attributed to the fact that the simulation only took the linear elasticity of the material into account. The accuracy of the simulation could be improved by considering a hyperelastic behavior for the carbon rod. Thus, to perform a nonlinear FE simulation, the mechanical properties of the carbon lever have to be characterized.

Figure 9 summarizes the stiffness variation with the length of the lever $\lambda$. The blue and red curves illustrate a model fitting done for the equations given by

$$
K_{\theta}=\frac{k_{0}}{\lambda}
$$

and

$$
K_{\theta}=\frac{k_{1}+k_{2} \lambda+k_{3} \lambda^{2}}{\lambda^{3}},
$$

where, $k_{0}, k_{1}, k_{2}$ and $k_{3}$ are the model parameters. Equations (15) and (16) are denoted model 1 and model 2 respectively. The continuous curves relate to the experimental results fittings, whereas the dashed curves relate to the simulation results fittings. Table II gives the parameters obtained with a non linear least-squares solver for each model fitting. It can

TABLE II: Model fitting parameters

\begin{tabular}{ccrrl}
\hline \hline Model & Parameter & Experimental results & \multicolumn{2}{c}{ Simulation results } \\
model 1 & $k_{0}$ & $2.54 \times 10^{2}$ & $2.71 \times 10^{2}$ \\
\hline \multirow{3}{*}{ model 2 } & $k_{1}$ & $2.32 \times 10^{5}$ & $1.54 \times 10^{6}$ \\
& $k_{2}$ & $-1.9 \times 10^{3}$ & $-2.22 \times 10^{2}$ \\
& $k_{3}$ & $6.15 \times 10^{2}$ & $2.53 \times 10^{2}$ \\
\hline \hline
\end{tabular}

be noticed that the fitted functions respect the order of the polynomial fraction obtained in (6) and (13) respectively. As 
one can see, the model 2 is more accurate to describe the angular stiffness behavior of the probe obtained during the experiment. Concerning the simulation results, both models describe the behavior observed, but it can be noticed that the coefficients obtained $k_{1}$ and $k_{2}$ are very small. This implies that model 2 is equivalent to the model 1 . Table III shows the sum of squared residuals for each curve fitting. This

TABLE III: Sums of squared residuals

\begin{tabular}{c|cc}
\hline \hline Data type & Experimental results & Simulation results \\
\hline Model & 0.9737 & 0.0242 \\
model 1 & 0.0205 & 0.0140 \\
\hline \hline
\end{tabular}

table shows that for the experimental and simulation results, the second model is more accurate than the first one. By comparing the sum of squared residuals obtained for the model 1 fitting with the experimental data with the other values, it confirms the model 1 is not suitable to accurately explain the behavior of the probes's stiffness.

The previous results show that model 2 is a good candidate to model the relation between the angular stiffness of the probe and the active length of the carbon rod. However, it has to be noticed that this model has some validity limits. First of all, it is valid only for small angles $\theta$. The second limit is the linearization of $M_{31}$, indeed, as Fig. 8 shows, the behavior is nonlinear for short active lengths of the carbon rod. Therefore computing the behavior of the equivalent stiffness of the probe from the linear regression of the torque $M_{31}$ induces some errors. The last limit concerns the assumption of a constant $c$ parameter. In the model presented, the point $O_{0^{\prime}}$ is assumed to be fixed. In reality, due to the play between the Base link and the carbon rod to allow the lever length to be changed, the position of $O_{0^{\prime}}$ depends on $\theta$ and $\lambda$.

The fitted function obtained with the second model monotonically decreases on the range of active length of carbon rod considered in this study. Thus the inverse of that function can be computed to define the lever length to obtain a desired angular stiffness of the probe. This method will be used in the future to design a stiffness controller for the probe.

Finally, Fig. 10 shows the first results of the VLM probe during a palpation on a silicone phantom with a stiff inclusion as described in section II-C and shown on Fig. 3. Furthermore, it shows the force and tactile sensor signal for three different stiffnesses level. Indeed, between each palpation, the stiffness of the probe have been changed $K_{\theta}=0.65,0.73$ and 0.84 $\mathrm{Nm} / \mathrm{rad}$ respectively. One can see that peaks on the force and some taxels signals are present when the probe slides over the stiff inclusion. The second part of the supplementary video shows the behavior of the VLM probe and the raw data measured by the sensors during the palpation test.

\section{DISCUSSION}

In this paper, only a static approach has been considered to model the novel VLM probe. However, force data in Fig. 7 show that there is some dynamic transient effect when a displacement is applied. Indeed those dynamic effects are
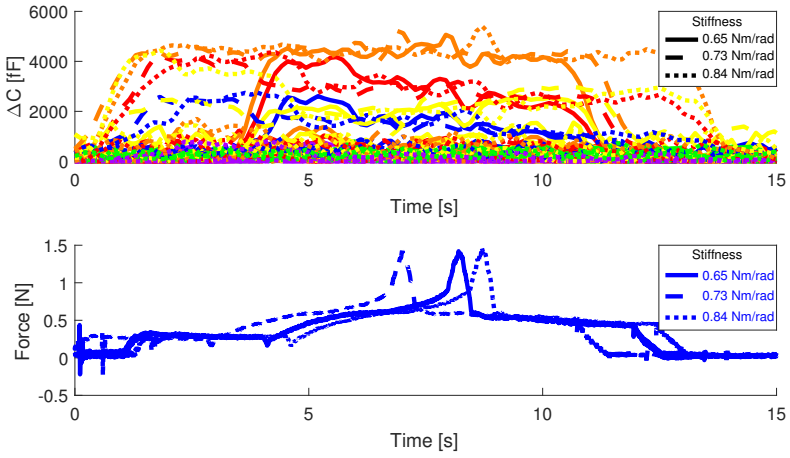

Fig. 10: Force and tactile signals measured during a test on a silicone phantom with a stiff inclusion. The different colors on the top figure refer to the different taxels.

related to the damping of the VLM which will be studied in the future. This will shed light on how the stiffness of the palpation probe influences tactile sensing and proprioceptive sensing (from the force sensor) during soft tissue palpation.

The majority of the VSAs and VSJs needs to be designed for a particular application. Indeed, since the size of the links has to be adapted to the robot, the range of stiffness variation is, most of the time, limited and the passive stiffness and damping components have to be selected in accordance with the expected behavior, limiting the availability of standard commercialized VSA. The aim of the given models is to help to design the probe and select the stiffness range by changing the mechanical properties of the lever as the young modulus or the second moment of area (by changing the diameter or the cross-section ) or the lever length. As discussed in section $\mathrm{V}$, even if the $c$ parameter, which defines the projected distance between the joint's rotation center and the clamped point of the carbon rod, has an impact, this parameter is not easy to identify due to some functional plays between parts.

Some other VSA designs implement a VLM principle for controlling the stiffness of the system. For example, Morita and Sugano proposed a VLM mechanism for a robotic finger with a lead screw, a slider, a leaf spring and a cable called the mechanical compliance adjuster [25]. This Variable Stiffness Joint (VSJ) has a stiffness range from $0.59 \mathrm{Nm} / \mathrm{rad}$ to $3.12 \mathrm{Nm} / \mathrm{rad}$ for a lever length from $0 \mathrm{~mm}$ to $40 \mathrm{~mm}$. Furthermore, it has a comparable length to the VLM probe proposed in this paper. However, the proposed solution has less volume and the weight of the mechanical compliance adjuster. Another important difference between the mechanical compliance adjuster and the mechanism described in this paper concerns the design approach. For the former, variable stiffness is obtained using rigid parts which are translating to change the boundary conditions, whereas, in the proposed VLM probe, it is the passive elastic component which is sliding. Finally, according to the authors' knowledge, the mechanical compliance adjuster has been tested for a few lever length and the relation to compute the lever length, needed to obtain a desired stiffness, have not been given. The proposed VLM probe design approach leads to an invertible function which gives the length of the flexible sliding lever to obtain a desired angular stiffness level of the probe. 
The results obtained during the palpation test clearly shows that the VLM probe can be used to detect stiff inclusion in soft tissue during a palpation. In future works, the authors will investigate the performances of the VLM probe for abdominal palpation to detect organ abnormalities and size/depth estimation of the organs. Indeed, based on our previous work on information gain maximization to detect hard nodule, the authors want to extend the method to an organ detection. Thus, a future implementation would follow a similar strategy by implementing an autonomous stiffness control which optimizes the information gain with a possible haptic feedback to help the user to determine the position of the organs. Also, the addition of the tactile sensor to the probe allows the author to investigate on the role of the tactile and force sensing during a palpation task, in order to better understand the physician gesture and the link between perception and action during palpation task.

\section{CONCLUSION}

This paper presents a new design of a variable stiffness palpation probe based on a variable lever mechanism (VLM). The experimental study shows that the new design has achieved an angular stiffness range from 0.64 N.m/rad to $1.06 \mathrm{~N} . \mathrm{m} / \mathrm{rad}$.

Two mechanical models have been proposed to describe the stiffness variation of the probe in response to varying carbon rod lever length using the Euler-Bernoulli beam theory. We found that the second model is more accurate to represent the non linear angular stiffness of the probe against the active length of the carbon rod.

An FE simulation has been performed to evaluate the range of the stiffness of the probe. The simulation results obtained have been compared to the experimental results and the results give a rough estimate of the real behavior. Some guidelines have been given to improve the FE simulation using a non linear hyperelastic behavior. To improve the accuracy of the simulation a knowledge of specific mechanical properties of the carbon rod seems to be necessary.

\section{REFERENCES}

[1] R. L. Siegel, K. D. Miller, and A. Jemal, "Cancer statistics, 2017," CA: A Cancer Journal for Clinicians, vol. 67, no. 1, pp. 7-30, 2017. [Online]. Available: http://dx.doi.org/10.3322/caac.21387

[2] C. Pacchierotti, D. Prattichizzo, and K. J. Kuchenbecker, "Cutaneous feedback of fingertip deformation and vibration for palpation in robotic surgery," IEEE Transactions on Biomedical Engineering, vol. 63, no. 2, pp. 278-287, Feb 2016

[3] N. Sornkarn, P. Dasgupta, and T. Nanayakkara, "Morphological computation of haptic perception of a controllable stiffness probe," PLOS ONE, vol. 11, no. 6, pp. 1-21, 062016.

[4] A. Talasaz and R. V. Patel, "Telerobotic palpation for tumor localization with depth estimation," in 2013 IEEE/RSJ International Conference on Intelligent Robots and Systems, Nov 2013, pp. 463-468.

[5] J. Konstantinova, A. Jiang, K. Althoefer, P. Dasgupta, and T. Nanayakkara, "Implementation of tactile sensing for palpation in robot-assisted minimally invasive surgery: A review," IEEE Sensors Journal, vol. 14, no. 8, pp. 2490-2501, Aug 2014.

[6] I. Kato, K. Kogenazawa, H. Fujimoto, and M. Hirata, "The automatic breast-cancer palpation robot : Wapro-4r," in IEEE International Workshop on Intelligent Robots, Oct 1988, pp. 73-78.

[7] V. Daniulaitis, M. O. Alhalabi, H. Kawasaki, and Y. Tanaka, "Medical palpation of deformable tissue using physics-based model for haptic interface robot (hiro)," in 2004 IEEE/RSJ International Conference on Intelligent Robots and Systems (IROS), vol. 4, Sept 2004, pp. 3907-3911 vol.4.
[8] S. Davaria, F. Najafi, M. J. Mahjoob, and S. M. Motahari-Bidgoli, "Design and fabrication of a robotic tactile device for abdominal palpation," in 2014 Second RSI/ISM International Conference on Robotics and Mechatronics (ICRoM), Oct 2014, pp. 339-344.

[9] A. L. Brazil, A. Conci, E. Clua, L. K. Bittencourt, and L. B. Baruque, "A virtual environment for breast exams practice with haptics and gamification," in 2017 IEEE 5th International Conference on Serious Games and Applications for Health (SeGAH), April 2017, pp. 1-7.

[10] M. Li, T. Ranzani, S. Sareh, L. D. Seneviratne, P. Dasgupta, H. A. Wurdemann, and K. Althoefer, "Multi-fingered haptic palpation utilizing granular jamming stiffness feedback actuators," Smart Materials and Structures, vol. 23, no. 9, p. 095007, 2014.

[11] L. Meli, C. Pacchierotti, and D. Prattichizzo, "Experimental evaluation of magnified haptic feedback for robot-assisted needle insertion and palpation," The International Journal of Medical Robotics and Computer Assisted Surgery, vol. 13, no. 4, p. e1809, 2017.

[12] N. Herzig, R. Moreau, A. Leleve, and M. T. Pham, "Stiffness control of pneumatic actuators to simulate human tissues behavior on medical haptic simulators," in 2016 IEEE International Conference on Advanced Intelligent Mechatronics (AIM), July 2016, pp. 1591-1597.

[13] N. Hogan, "Stable execution of contact tasks using impedance control," in Robotics and Automation. Proceedings. 1987 IEEE International Conference on, vol. 4, Mar 1987, pp. 1047-1054.

[14] N. Herzig, R. Moreau, T. Redarce, F. Abry, and X. Brun, "Non linear position and closed loop stiffness control for a pneumatic actuated haptic interface: the BirthSIM," in IEEE/RSJ International Conference on Intelligent Robots and Systems (IROS), Hamburg, Sept 2015, pp. $1612-1618$

[15] T. Nanayakkara, A. Jiang, M. del Roco Armas Fernndez, H. Liu, K. Althoefer, and J. Bimbo, "Stable grip control on soft objects with time-varying stiffness," IEEE Transactions on Robotics, vol. 32, no. 3, pp. 626-637, June 2016.

[16] B. Vanderborght, A. Albu-Schaeffer, A. Bicchi, E. Burdet, D. Caldwell, R. Carloni, M. Catalano, O. Eiberger, W. Friedl, G. Ganesh, M. Garabini, M. Grebenstein, G. Grioli, S. Haddadin, H. Hoppner, A. Jafari, M. Laffranchi, D. Lefeber, F. Petit, S. Stramigioli, N. Tsagarakis, M. V. Damme, R. V. Ham, L. Visser, and S. Wolf, "Variable impedance actuators: A review," Robotics and Autonomous Systems, vol. 61, no. 12, pp. $1601-1614,2013$.

[17] R. Van Ham, T. Sugar, B. Vanderborght, K. Hollander, and D. Lefeber, "Compliant actuator designs," IEEE Robotics Automation Magazine, vol. 16, no. 3, pp. 81-94, September 2009.

[18] X. Huo, Y. Xia, Y. Liu, L. Jiang, and H. Liu, "Humanoid arm with the integrated serial elastic actuator," in 2014 IEEE International Conference on Robotics and Biomimetics (ROBIO 2014), Dec 2014, pp. 2321-2326.

[19] R. Schiavi, G. Grioli, S. Sen, and A. Bicchi, "VSA-II: a novel prototype of variable stiffness actuator for safe and performing robots interacting with humans," in 2008 IEEE International Conference on Robotics and Automation, May 2008, pp. 2171-2176.

[20] K. W. Hollander, T. G. Sugar, and D. E. Herring, "Adjustable robotic tendon using a 'jack spring'," in 9th International Conference on Rehabilitation Robotics, 2005. ICORR 2005., June 2005, pp. 113-118.

[21] R. Furnmont, G. Mathijssen, T. van der Hoeven, B. Brackx, D. Lefeber, and B. Vanderborght, "Torsion maccepa: A novel compact compliant actuator designed around the drive axis," in 2015 IEEE International Conference on Robotics and Automation (ICRA), May 2015, pp. 232237.

[22] N. Sornkarn, P. Dasgupta, and T. Nanayakkara, "Morphological computation of haptic perception of a controllable stiffness probe," PloS one, vol. 11, no. 6, p. e0156982, 2016.

[23] M. I. Awad, D. Gan, M. Cempini, M. Cortese, N. Vitiello, J. Dias, P. Dario, and L. Seneviratne, "Modeling, design characterization of a novel passive variable stiffness joint (pVSJ)," in 2016 IEEE/RSJ International Conference on Intelligent Robots and Systems (IROS), Oct 2016, pp. 323-329.

[24] T. H. L. Le, P. Maiolino, F. Mastrogiovanni, and G. Cannata, "Skinning a robot: Design methodologies for large-scale robot skin," IEEE Robotics Automation Magazine, vol. 23, no. 4, pp. 150-159, Dec 2016.

[25] T. Morita and S. Sugano, "Design and development of a new robot joint using a mechanical impedance adjuster," in Proceedings of 1995 IEEE International Conference on Robotics and Automation, vol. 3, May 1995, pp. 2469-2475 vol.3. 